\title{
Generation of a Nearly-Monocycle Optical Pulse in the Near- Infrared Region and Its Use as an Ionization Source in Mass Spectrometry
}

\author{
Supporting information
}

\author{
Yuta Nakano, ${ }^{\dagger}$ Tomoko Imasaka, ${ }^{\S, *}$ Totaro Imasaka ${ }^{\ddagger, \#}$ \\ ${ }^{\dagger}$ Department of Applied Chemistry, Graduate School of Engineering, Kyushu University, 744 \\ Motooka, Nishi-ku, Fukuoka 819-0395, Japan \\ §Department of Environmental Design, Faculty of Design, Kyushu University, 4-9-1 Shiobaru, \\ Minami-ku, Fukuoka 815-8540, Japan \\ ‡Division of International Strategy, Center of Future Chemistry, Kyushu University, 744 Motooka, \\ Nishi-ku, Fukuoka 819-0395, Japan \\ \#Hikari Giken, Co., 2-10-30, Sakurazaka, Chuou-ku, Fukuoka 810-0024, Japan
}

* To whom correspondence should be addressed. E-mail: imasaka@design.kyushu-u.ac.jp 


\section{Table of contents:}

\section{Experimental Setup and Spectral Data}

Fig. S1. Experimental setup used for the generation and characterization of the NIR optical pulse.

Fig. S2. Optical properties of the chirp mirror at $800 \mathrm{~nm}$ (Thorlabs).

Fig. S3. Optical properties of the chirp mirror at $800 \mathrm{~nm}$ (Layertec).

Fig. S4. Optical properties of the chirp mirror set at $1200 \mathrm{~nm}$.

Fig. S5. Optical properties of the dichroic half mirror used for synthesizing the 800- and 1200-nm pulses.

Fig. S6. Experimental setup of the SHG FROG system.

Fig. S7. Spectral domain available for an SHG FROG system using a 5- $\mu$ m-thick BBO crystal.

Fig. S8. Experimental setup for the generation of a 600-nm optical pulse.

Fig. S9. Experimental setup for a feedback control to improve the long-term stability of the pulse shape.

Fig. S10. Experimental setup for THG of the 3.2-fs NIR optical pulse in Ar gas.

\section{Experimental Data}

Fig. S11. Spectrum of the pulse consisting of three colors and temporal profile calculated by assuming a transform-limited pulse.

Fig. S12. Phase lock of the pulses.

Fig. S13. Long-term stability of the spectrum measured at around $600 \mathrm{~nm}$ without and with a feedback control.

Fig. S14. Data obtained for a UV pulse.

Fig. S15. Spectrum synthesized using the data of the UV and VIS-NIR pulses and temporal profile calculated by assuming a transform-limited pulse.

\section{Mass Spectrometry}

Fig. S16. Calculated absorption spectra for pentachlorobenzene.

Fig. S17. Mass spectra for pentachlorobenzene measured using the 3.2-fs pulse.

\section{Novichok}

Fig. S18. Chemical structures for Mirzayanov's and non-Mirzayanov's Novichok.

Fig. S19. Absorption spectra calculated for Mirzayanov's Novichok.

Fig. S20. Absorption spectra calculated for non-Mirzayanov's Novichok.

\section{References}

Refs. S1-S8. 


\section{Experimental Setup and Spectral Data}

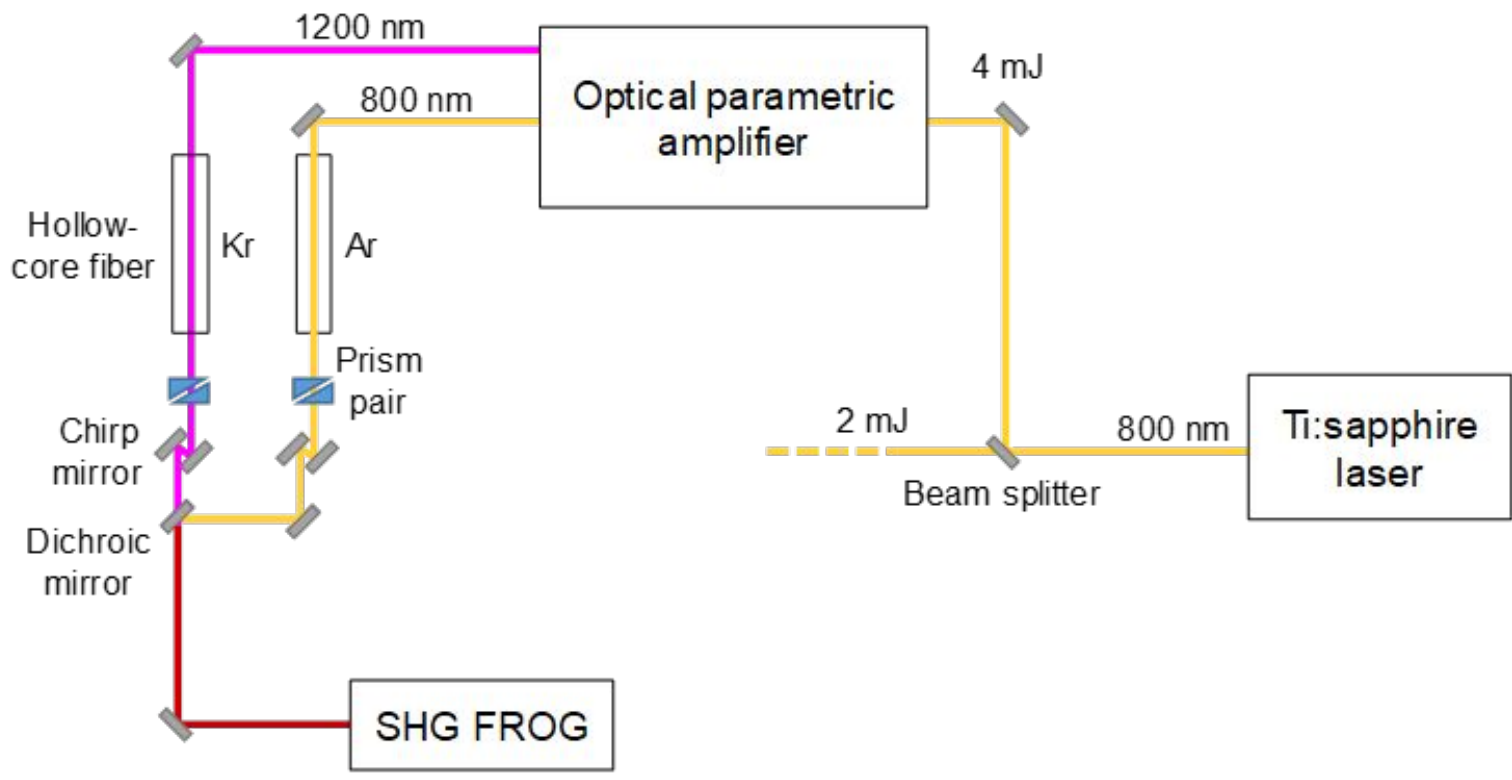

Fig. S1. Experimental setup used for the generation and characterization of the NIR optical pulse. The timing between the two pulses at 800 and $1200 \mathrm{~nm}$ was changed by adjusting the position (not measurable) of the stage in the OPA, on which the mirror reflecting the 800 -nm beam was mounted.
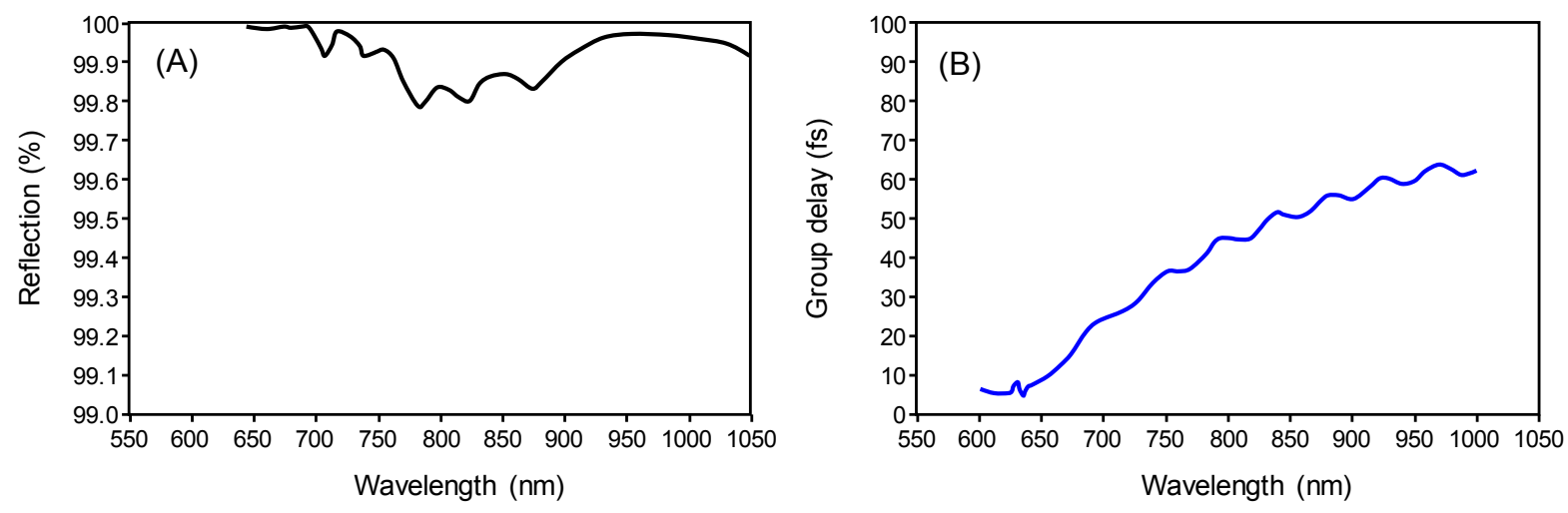

Fig. S2. Optical properties of the chirp mirror at $800 \mathrm{~nm}$ (Thorlabs). (A) reflection (B) group delay (GD). Data for group delay dispersion (GDD) were not available from the manufacturer. 

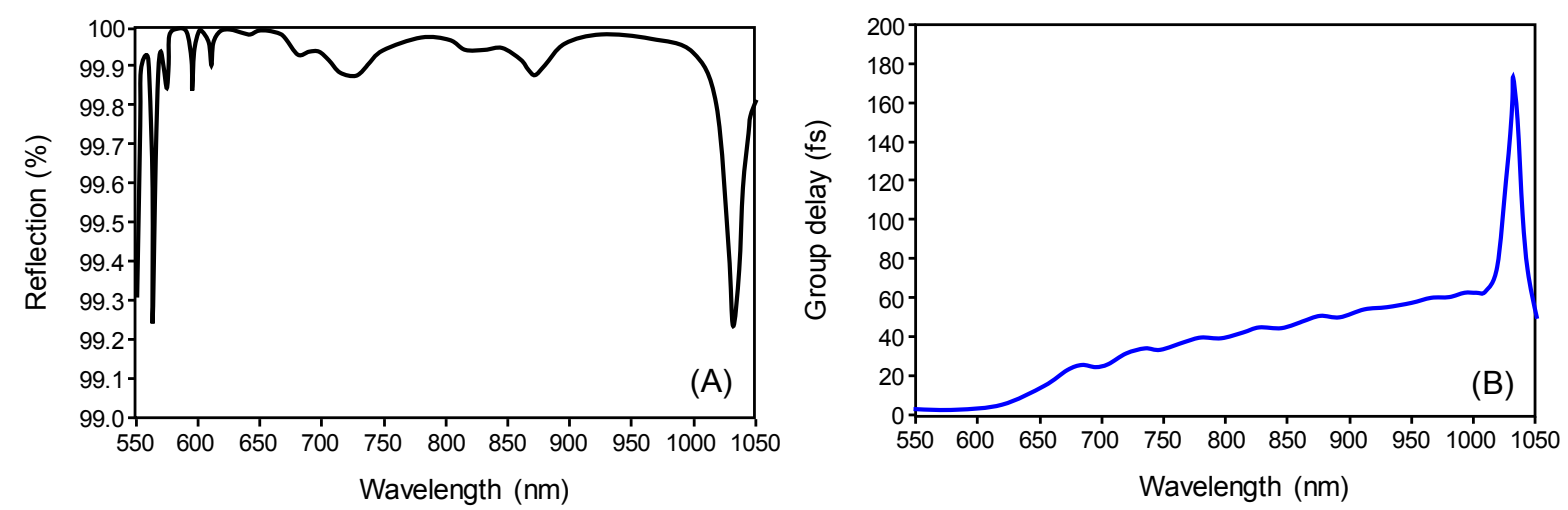

Fig. S3. Optical properties of the chirp mirror at $800 \mathrm{~nm}$ (Layertec). (A) reflection (B) GD. Data for GDD were not available from the manufacturer.
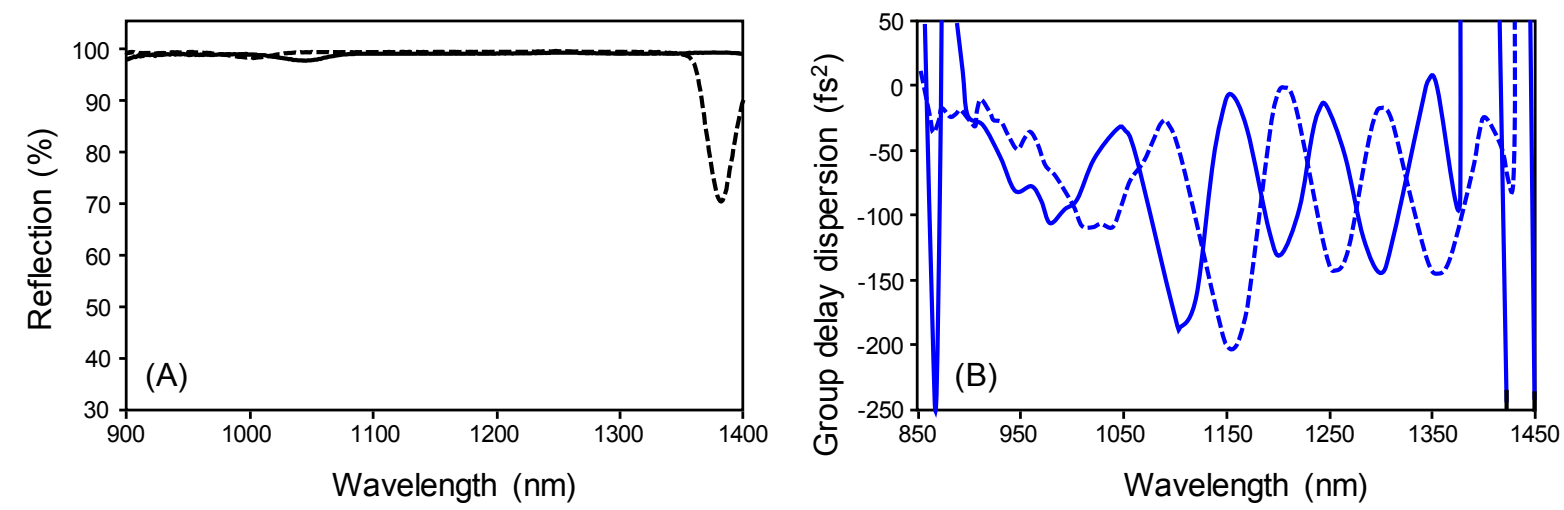

Fig. S4. Optical properties of the chirp mirror set at $1200 \mathrm{~nm}$. (A) reflection (B) GDD.
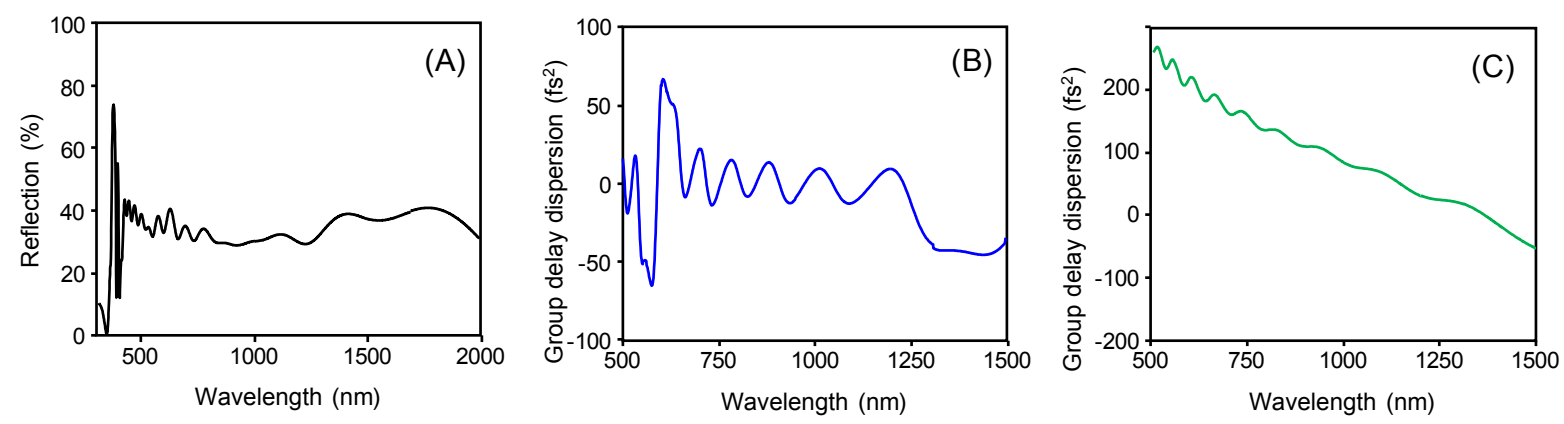

Fig. S5. Optical properties of the dichroic half mirror used for synthesizing the 800- and 1200-nm pulses. (A) reflection (B) GDD for reflection, (C) GDD for transmission. The main part of the GDD for transmission arises from the substrate material of the mirror. Note that the value for the GDD is wavelength-dependent, suggesting the third-order dispersion to be compensated for better pulse compression. 


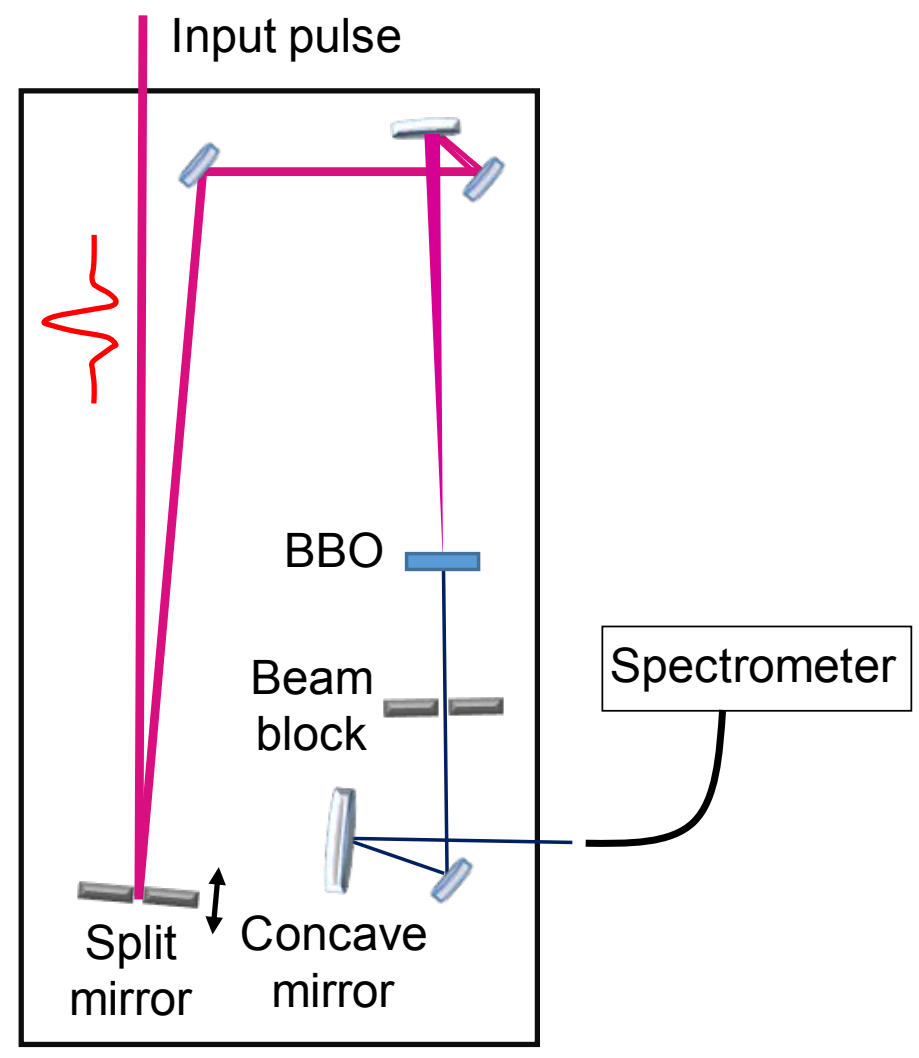

Fig. S6. Experimental setup of the SHG FROG system. Note that the spectral band width of SHG FROG is limited to one octave. Validity of the FROG measurement can be judged from the FROG error whether the value is less than $1 \%$ or not.

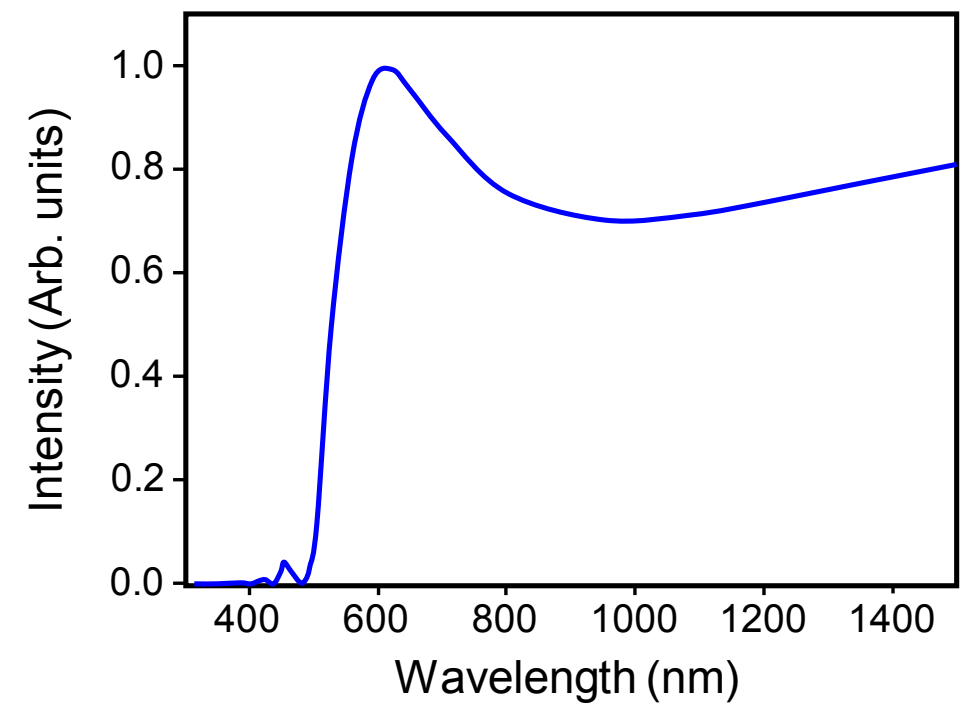

Fig. S7. Spectral domain available for an SHG FROG system using a 5- $\mu$ m-thick BBO crystal, suggesting that the system can be used for the characterization of the pulse in the spectral region of 550-1500 nm. 


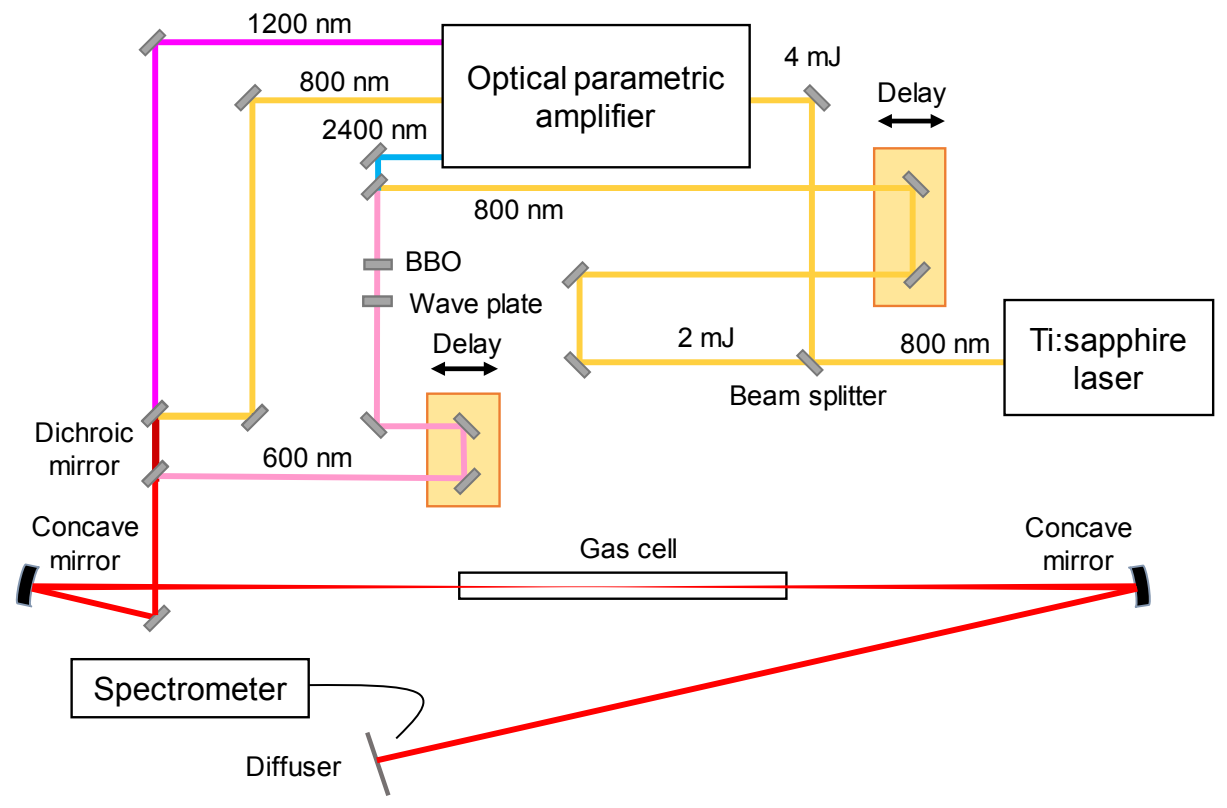

Fig. S8. Experimental setup for the generation of a $600-\mathrm{nm}$ optical pulse by sum frequency mixing of the 800- and 2400-nm pulses and for the measurement of the stability of the relative phase against the first Stokes emission $(600 \mathrm{~nm})$ generated by FWRM of the 800- and 1200-nm pulses in $\mathrm{H}_{2}(0.1 \mathrm{MPa})$ in a gas cell. The width of the 600 -nm pulse was determined to be $30 \mathrm{fs}$ using the SHG FROG system (FROG error, 0.8\%).

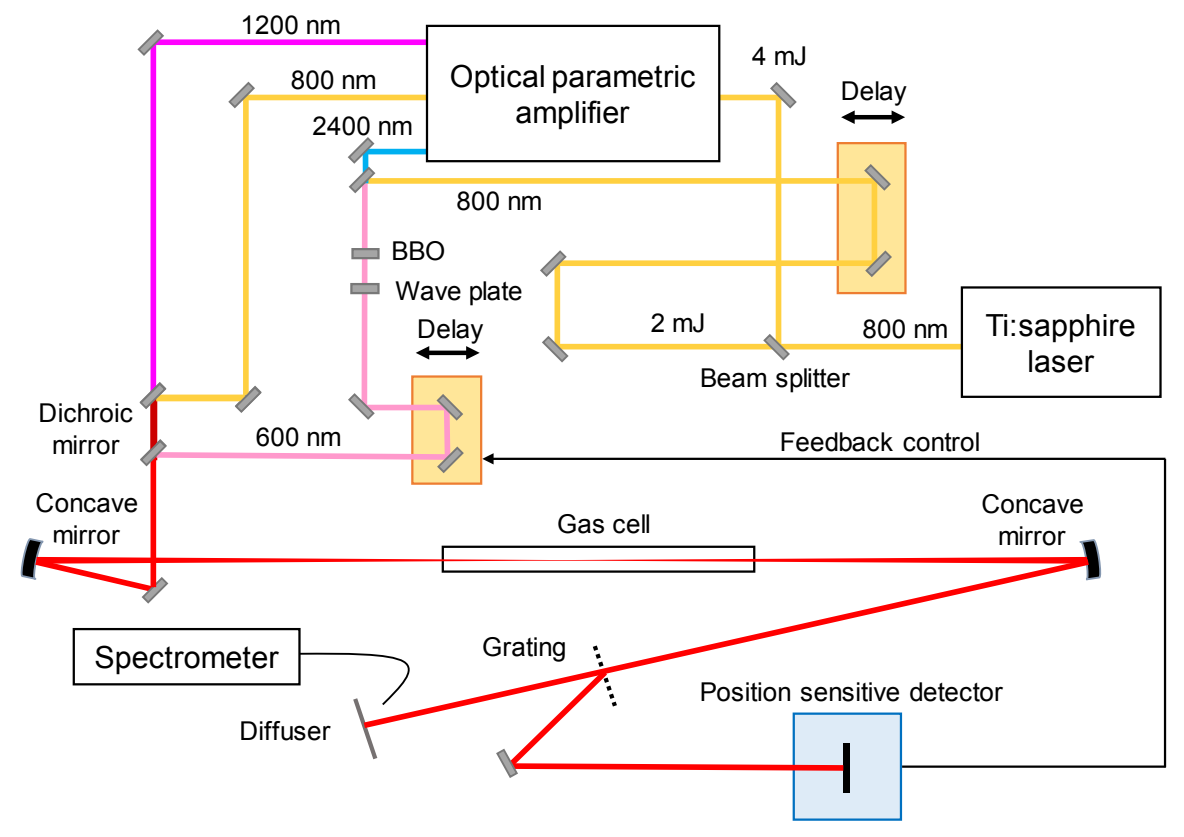

Fig. S9. Experimental setup for a feedback control to improve the long-term stability of the pulse shape by superimposing the $600-\mathrm{nm}$ pulse generated by sum frequency mixing of the 800 - and $2400-\mathrm{nm}$ pulses with the $600-\mathrm{nm}$ pulse generated by FWRM of the 800 - and 1200 -nm pulses. 


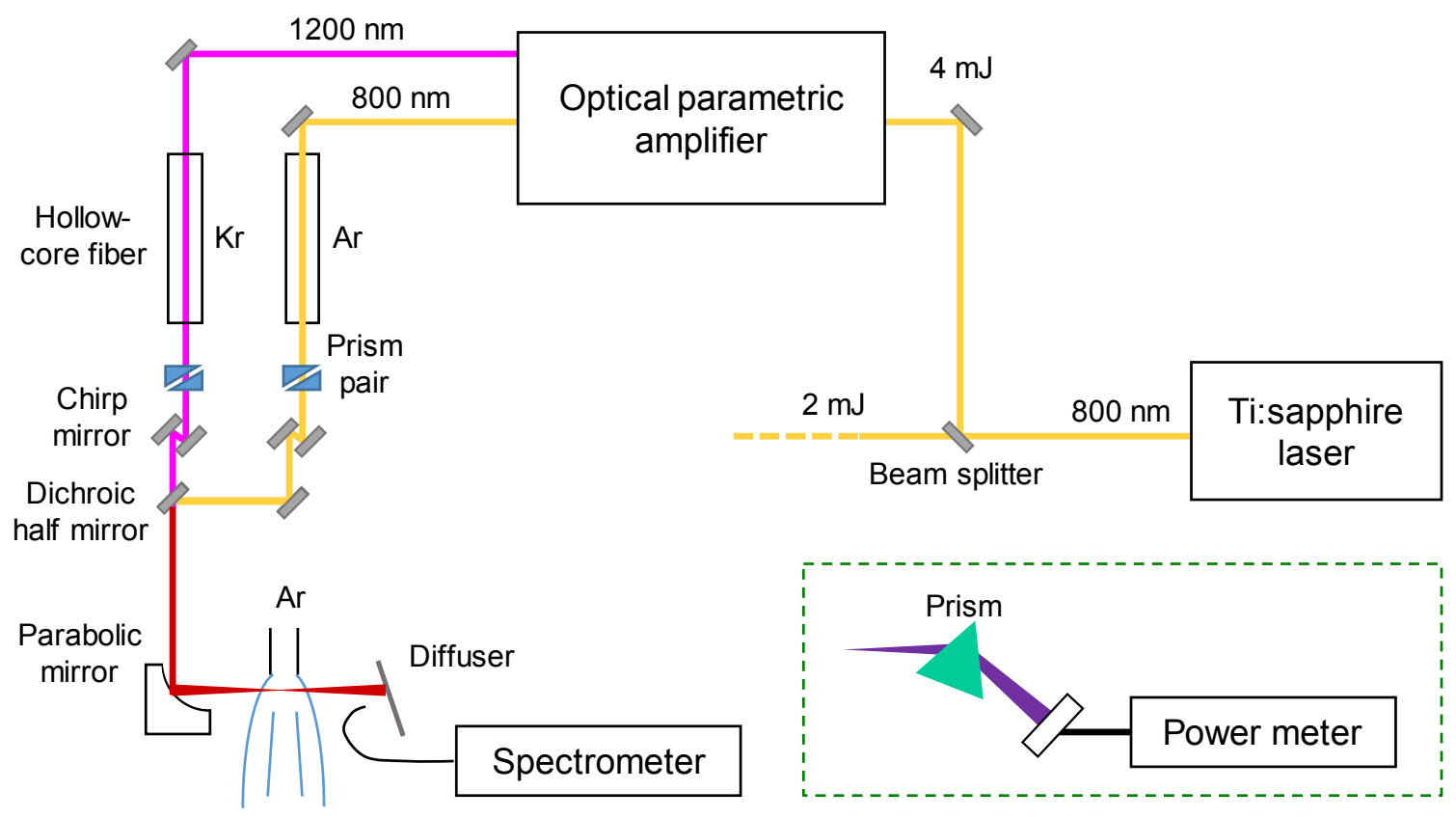

Fig. S10. Experimental setup for THG of the 3.2-fs NIR optical pulse in Ar gas.

\section{Experimental Data}
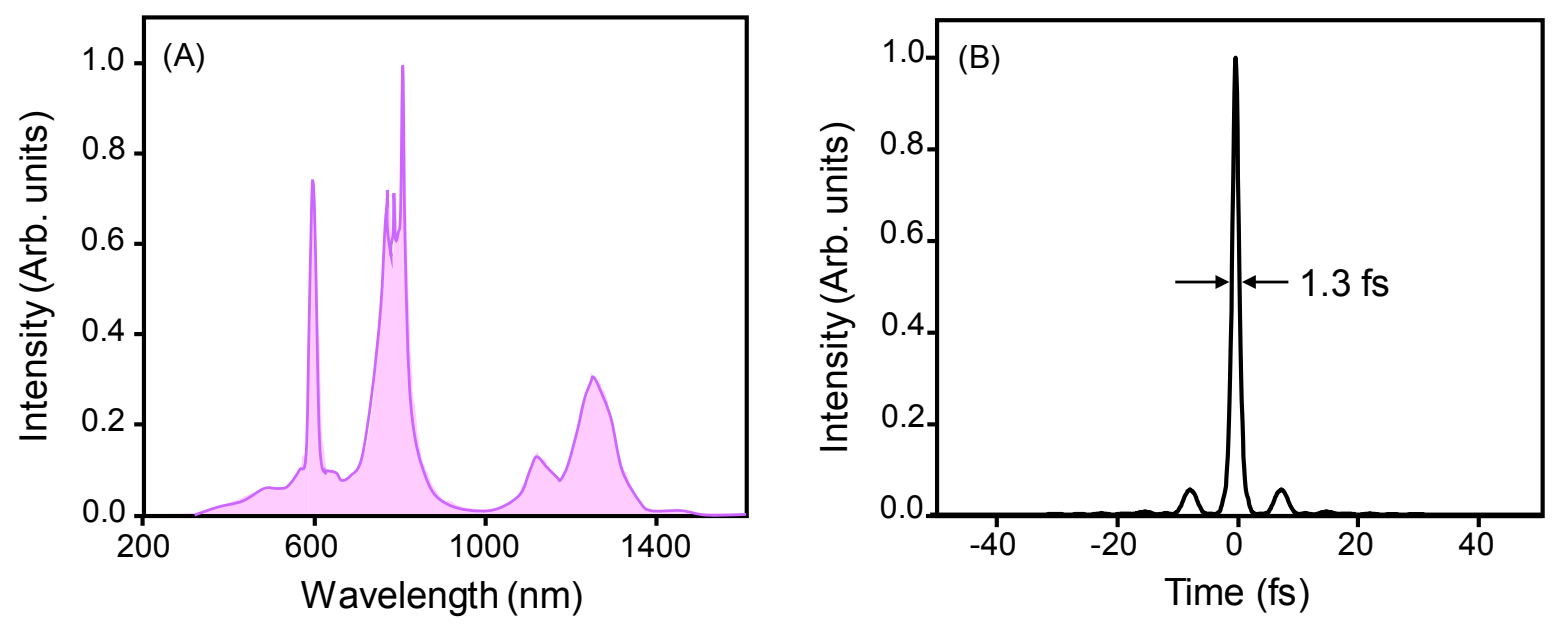

Fig. S11. (A) spectrum of the pulse consisting of three colors $(600,800,1200 \mathrm{~nm})$. (B) temporal profile calculated from data (A) by assuming a transform-limited pulse. The spectrum was measured by focusing the three-color beam onto Xe (0.2 MPa) in the gas cell shown in Fig. S8. 

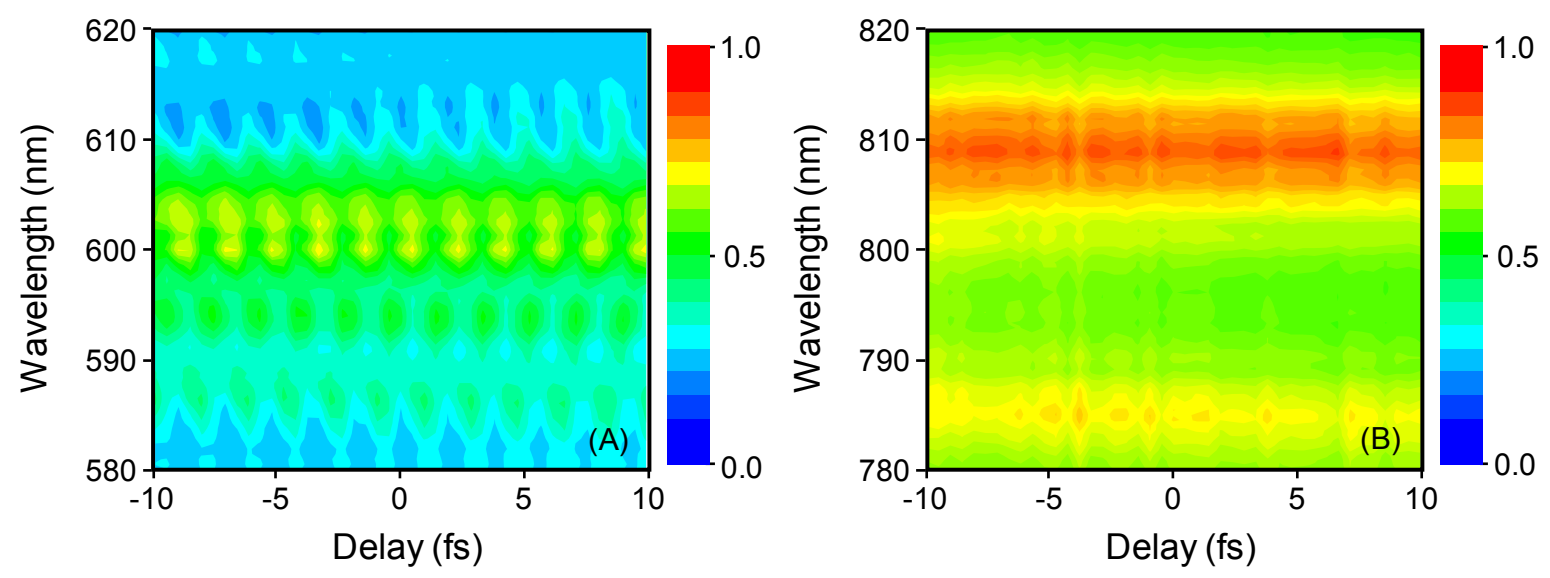

Fig. S12. Phase lock of the pulses. (A) spectrogram obtained by changing the delay of the $600-\mathrm{nm}$ pulse generated by sum frequency mixing of the 800 - and 2400 -nm pulses against the $600-\mathrm{nm}$ pulse generated by FWRM of the 800 - and 1200-nm pulses. (B) spectrogram obtained by changing the delay of the fundamental TS pulse $(800 \mathrm{~nm})$ against the $800-\mathrm{nm}$ pulse generated by FWRM of the signal $(1200 \mathrm{~nm})$ and idler (2400 $\mathrm{nm})$ pulses. The periodic fringe pattern observed in (A) suggests that the relative phases of the pulses are locked and the change in the optical path lengths is minimal because of nearly identical optical geometry for the TS and OPA beams.
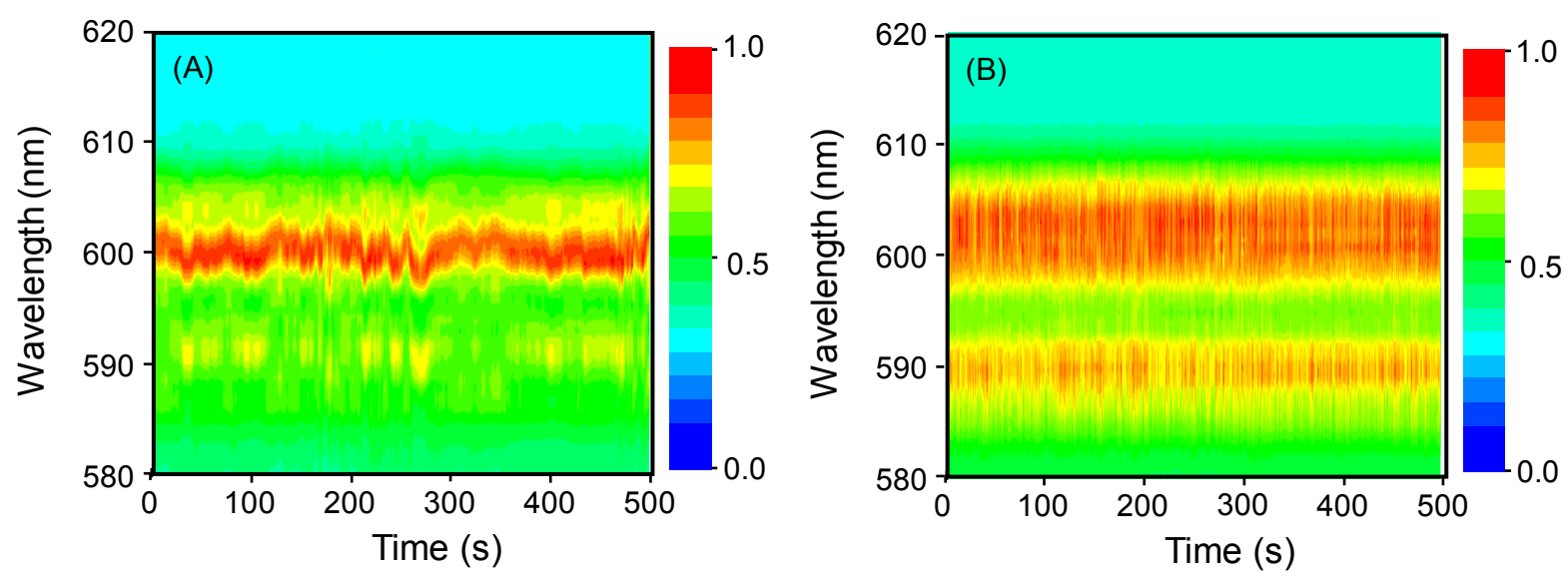

Fig. S13. Long-term stability of the spectrum measured at around $600 \mathrm{~nm}$ without (A) with (B) a feedback control. The spectral pattern is fixed in (B), suggesting the optical path length can be fixed by a feedback control. The high-frequency noise in (B) arose from the fast response time of the feedback system. The stability of the spectral pattern was also confirmed by on-line real-time monitoring of the spectrum using the spectrometer. Actually, the spectral peaks drifted in (A) but remained fixed in (B). It should be noted that poor stability in (A) arises from the different beam path of the 800-nm beam (see Fig. S8). The present system can be used for improving long-term stability in the experiment using the two-color beam (Fig. S12). 

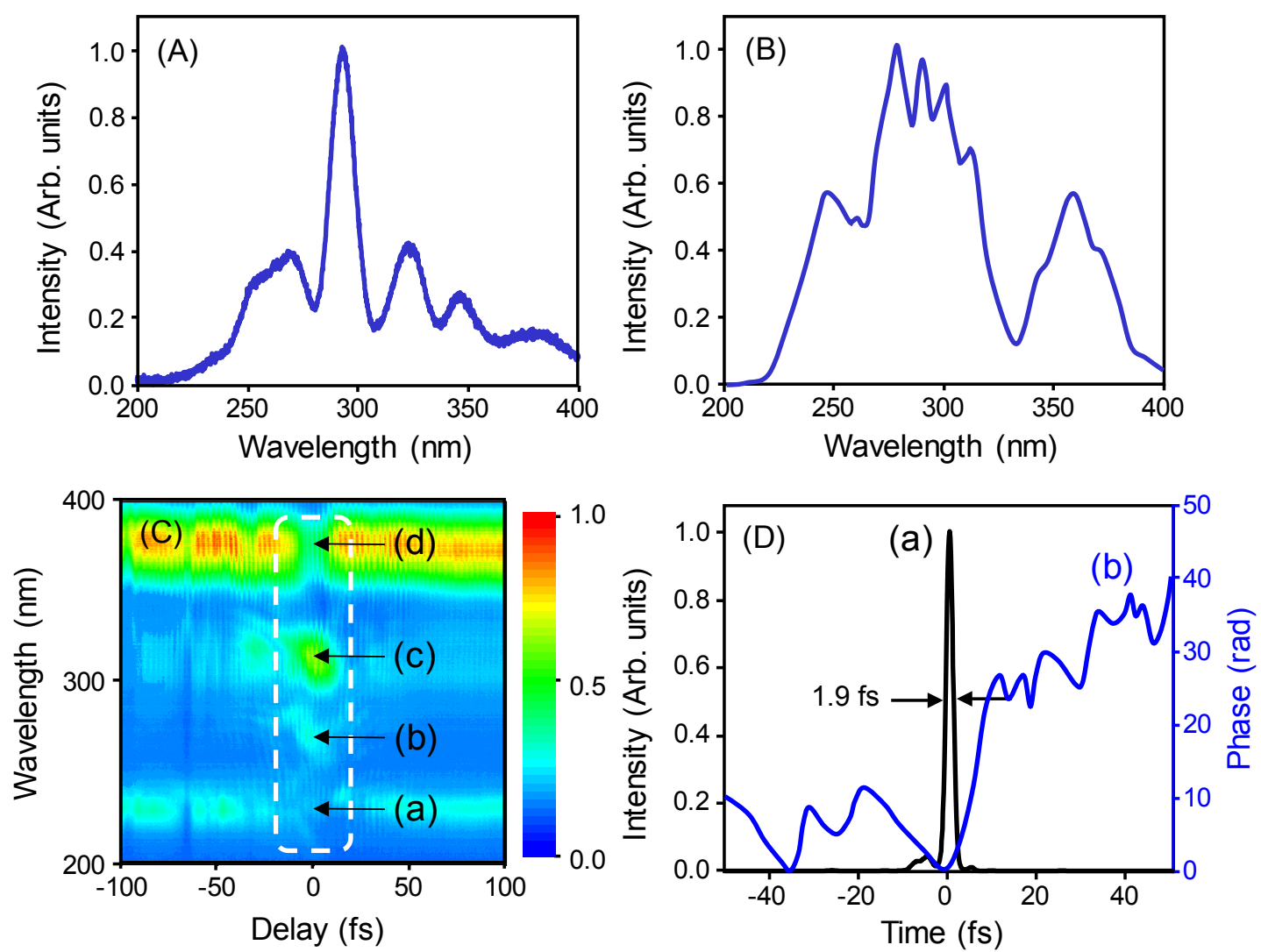

Fig. S14. Data obtained for a UV pulse. (A) observed spectrum (B) spectrum calculated from the data obtained by SHG FROG (C) spectrogram obtained by changing the delay of the 800 -nm pulse (D) temporal profile (a) and phase (b) calculated by assuming THG of the NIR pulse. The temporal profile of the transformlimited pulse (1.3 fs) is not shown in (D) to simplify the figure.
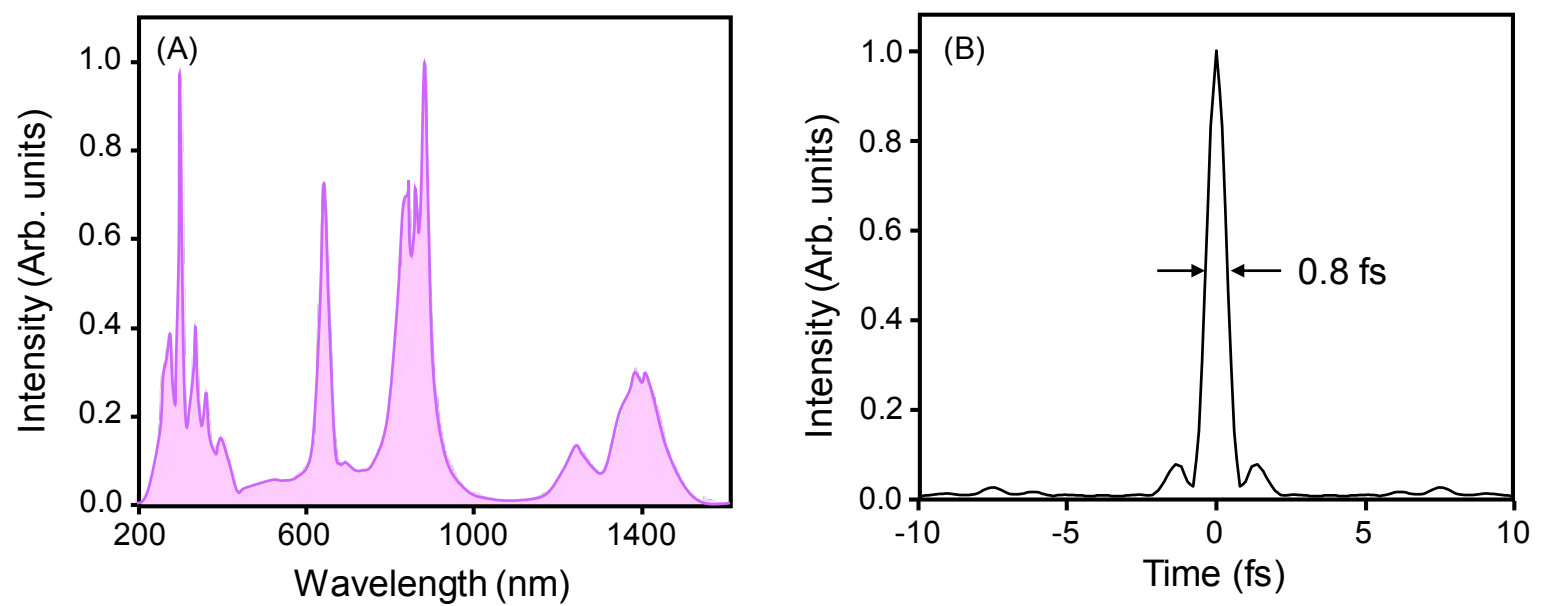

Fig. S15. (A) spectrum synthesized using the data of the UV and VIS-NIR pulses. The intensity of the VISNIR pulse was attenuated so as to be nearly equal to that of the UV pulse. (B) temporal profile calculated by assuming a transform-limited pulse. 


\section{Mass Spectrometry}

The Stockholm Convention on Persistent Organic Pollutants is an international environmental treaty, signed in 2001 and effective starting from May 2004, that aims to eliminate or restrict the production and use of persistent organic pollutants (POPs). They are defined as chemical substances that persist in the environment, bioaccumulate through the food web, and pose a risk of causing adverse effects to human health and the environment. ${ }^{\mathrm{S} 1}$ Twenty-nine organic compounds including pentachlorobenzene are specified as POPs in the convention.
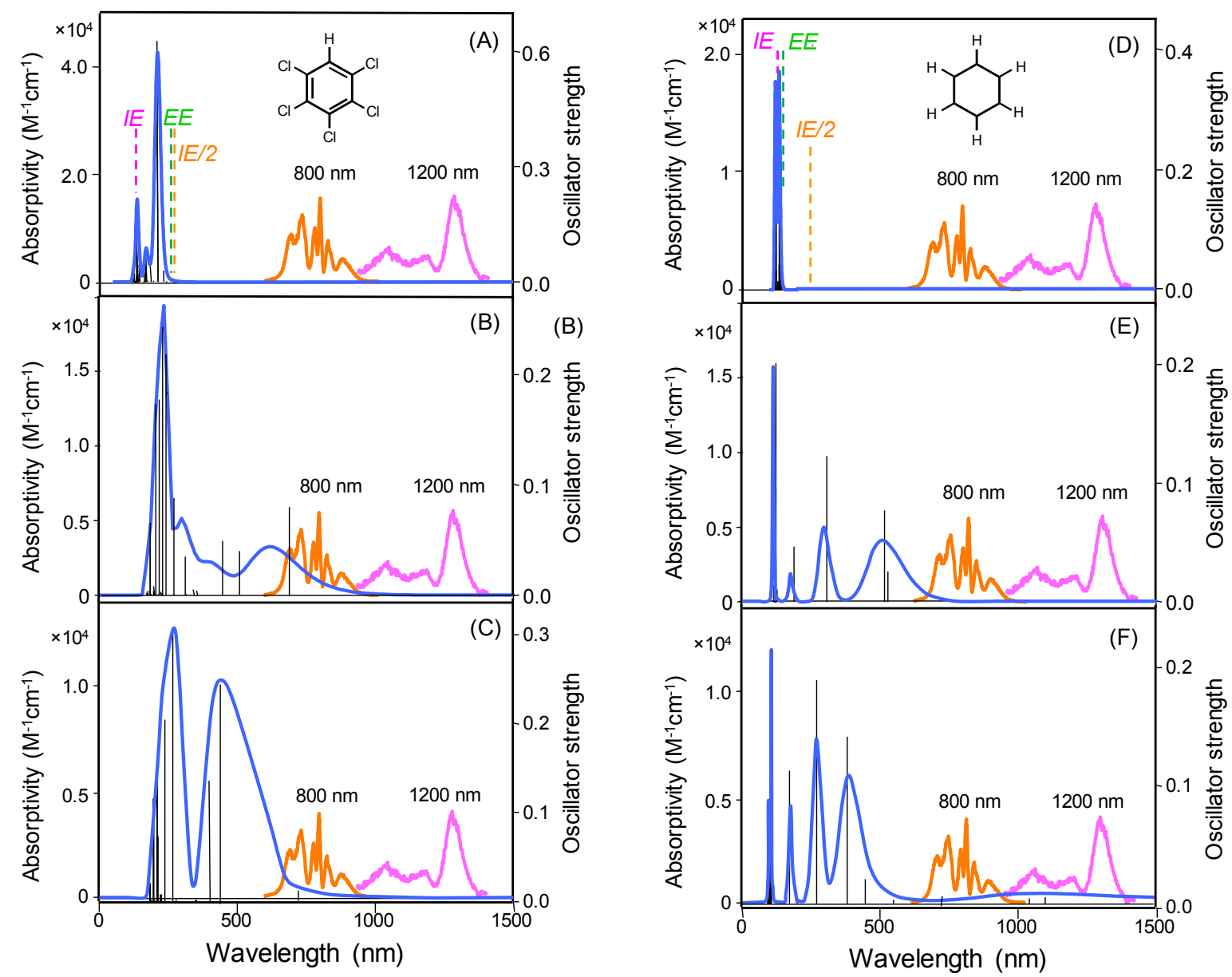

Fig. S16. Calculated absorption spectra for pentachlorobenzene (A) - (C) and cyclohexane (D) - (F) for neutral species (A) (D), a singly-charged ion (B) (E), and a doubly-charged ion (C) (F). The wavelengths corresponding to the first excited energy $(E E)$, the ionization energy $(I E)$, and the half value of the ionization energy $(I E / 2)$ are indicated in the figure. The emission spectrum of the ionization source is shown in the figure. 


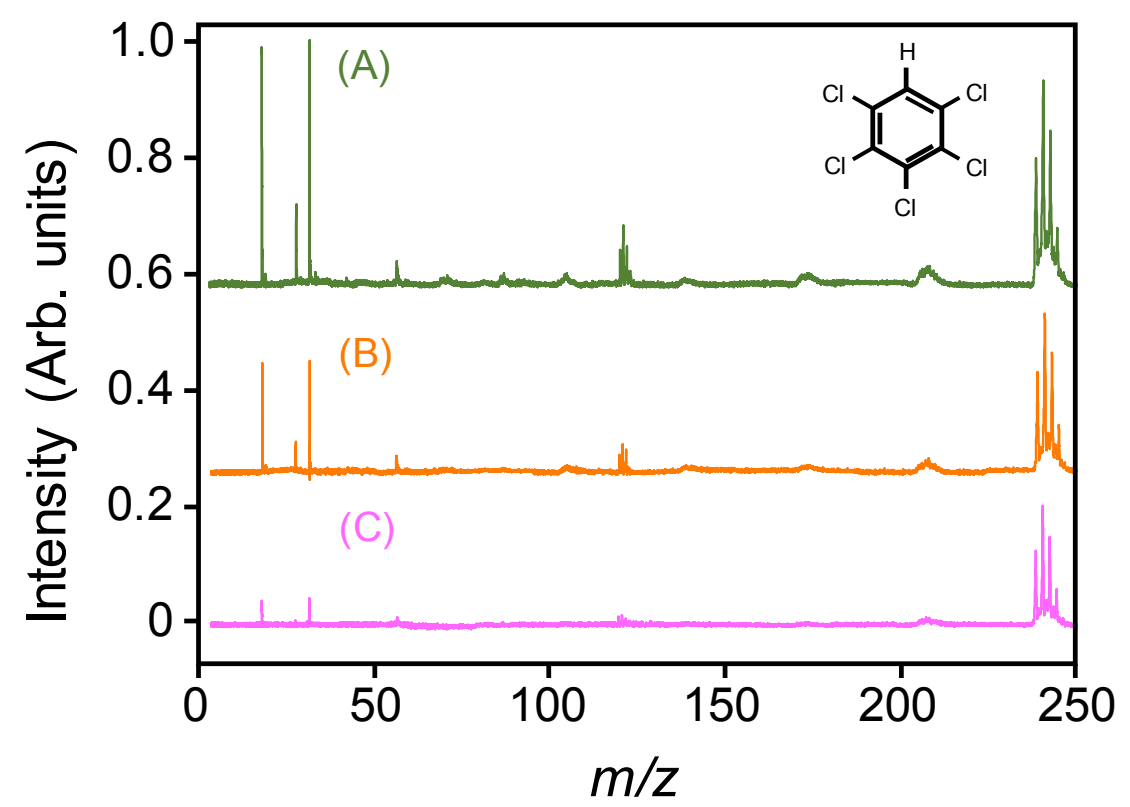

Fig. S17. Mass spectra for pentachlorobenzene measured using the 3.2-fs pulse. Pulse energy ( $\mu \mathrm{J})$, (A) 120 (B) 100 (C) 80. Keldysh parameter ( $\gamma$ ), (A) 0.20 (B) 0.22 (C) 0.25.

\section{Novichok}

On the 12th of March 2018, the UK government announced that a Novichok agent had been used in an attack in the English city of Salisbury on the 4th of March 2018 in an attempt to kill a former GRU officer Sergel Skripal and his daughter Yulia. ${ }^{\mathrm{S} 2 \mathrm{S3}}$ Because this nerve agent is extremely toxic and is outlawed worldwide, it is important to confirm that it was actually used in the attack and to identify the nerve agent and the criminal who used it.

Only limited information is currently available regarding the analysis of Novichok. However, gas chromatography combined with mass spectrometry (GC-MS) would be a potentially useful technique for achieving this, given the fact that an analyte can be separated by GC and then identified by MS. In this technique, it is necessary to collect data on a standard sample to determine the retention time in GC and the mass spectral pattern for identification prior to the analysis. Although the chemical formula of Novichok is known (Fig. S18), no standard samples are available (except for a specially-permitted or illegal laboratory), since the synthesis of this agent is prohibited by law. If a molecular ion as well as fragment ions could be measured, the analyte could readily be confirmed from a mass/charge ratio, i.e., its molecular weight in MS. However, a flexible nitrogencontaining aliphatic molecule seldom provides a molecular ion by electron ionization in MS as has been reported 
for drugs such as tryptamine and phenethylamine, ${ }^{\mathrm{S} 5}$ although Novichok derivatives (not Mirzayanov's Novichok) have been reported to provide a molecule ion. ${ }^{\mathrm{S} 6}$

Weapons-grade nerve agents are relatively impure and typically contain many types of byproducts that are produced in the manufacturing process. Thus, information concerning the constituents in the sample, which can be obtained by a comprehensive analysis based on GC-MS, could provide valuable information for the identification of the manufacturer. This technique provides a two-dimensional display of GC vs. MS, which can be used for the analysis of unknown chemical species. It should be noted here that metabolites derived from nerve agents have been measured using this method and subfemtogram detection limits (0.4-5.9 fg) have been achieved for polycyclic aromatic hydrocarbons, which is, to our knowledge, the lowest values reported in comprehensive GC-MS analyses. ${ }^{\mathrm{S} 7, \mathrm{~S} 8}$

(A)

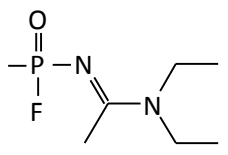

(D)

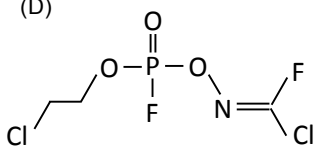

(B)

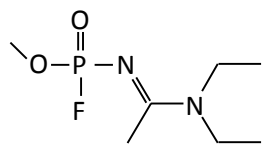

(E)

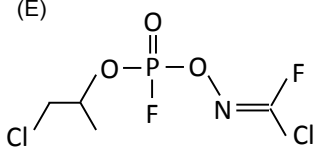

[. C)

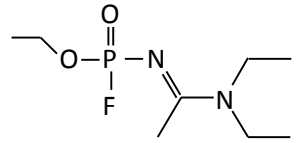

(F)<smiles>CC(Cl)C(C)OP(=O)(F)O/N=C(\F)Cl</smiles>

Fig. S18. Chemical structures. (A) - (C) Mirzayanov's (D) - (F) non-Mirzayanov's Novichok. ${ }^{\mathrm{S}}$ (A) (D) A230 (B) (E) A232 (C) (F) A234. Non-Mirzayanov's Novichok would be more dissociative than Mirzayanov's Novichok because of the ether groups in the molecule.
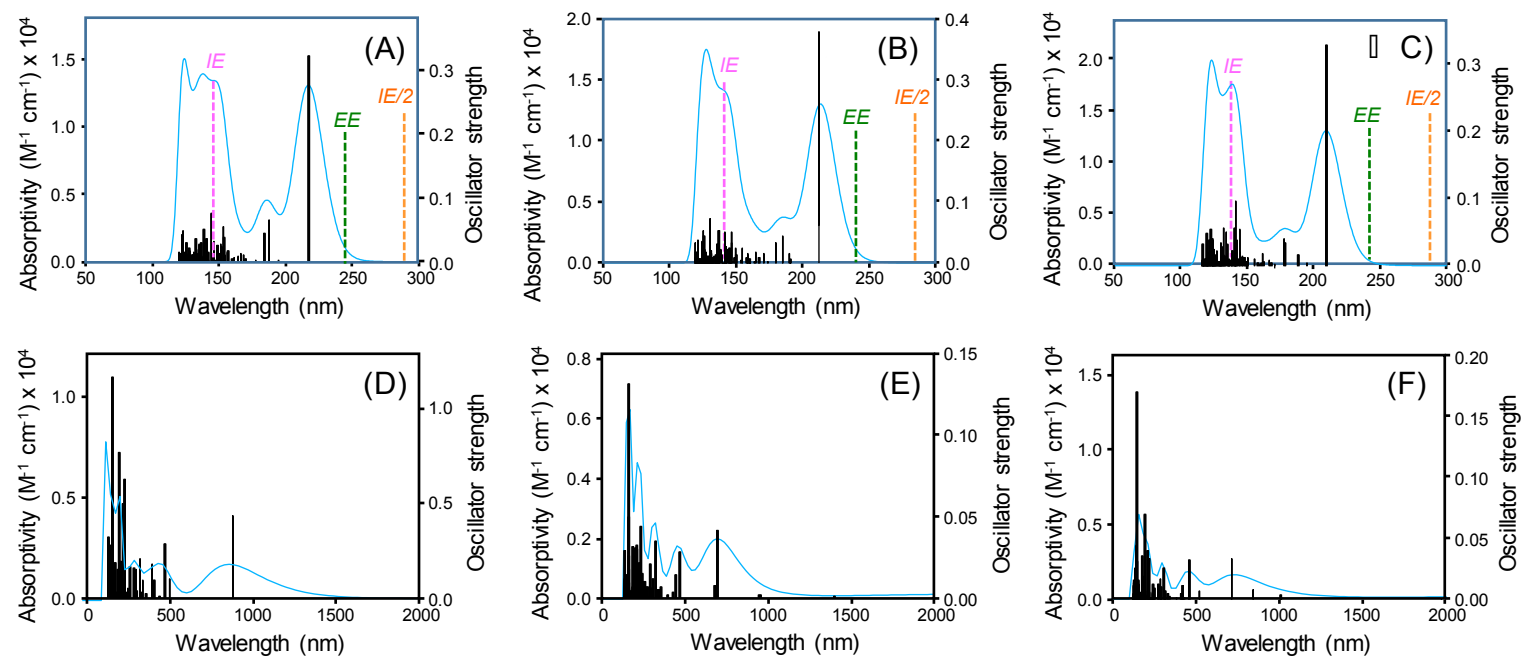

Fig. S19. Absorption spectra calculated for Mirzayanov's Novichok. (A) - (C) absorption spectra for neutral species. (D) - (F) absorption spectra for singly-charged ions. (A) (D) A230 (B) (E) A232 (C) (F) A234. The wavelengths corresponding to the first excited energies $(E E)$, the ionization energy $(I E)$, and the half value of the ionization energy (IE/2) are indicated in the figure. It is suspected that A232 was used in Salisbury, UK. 

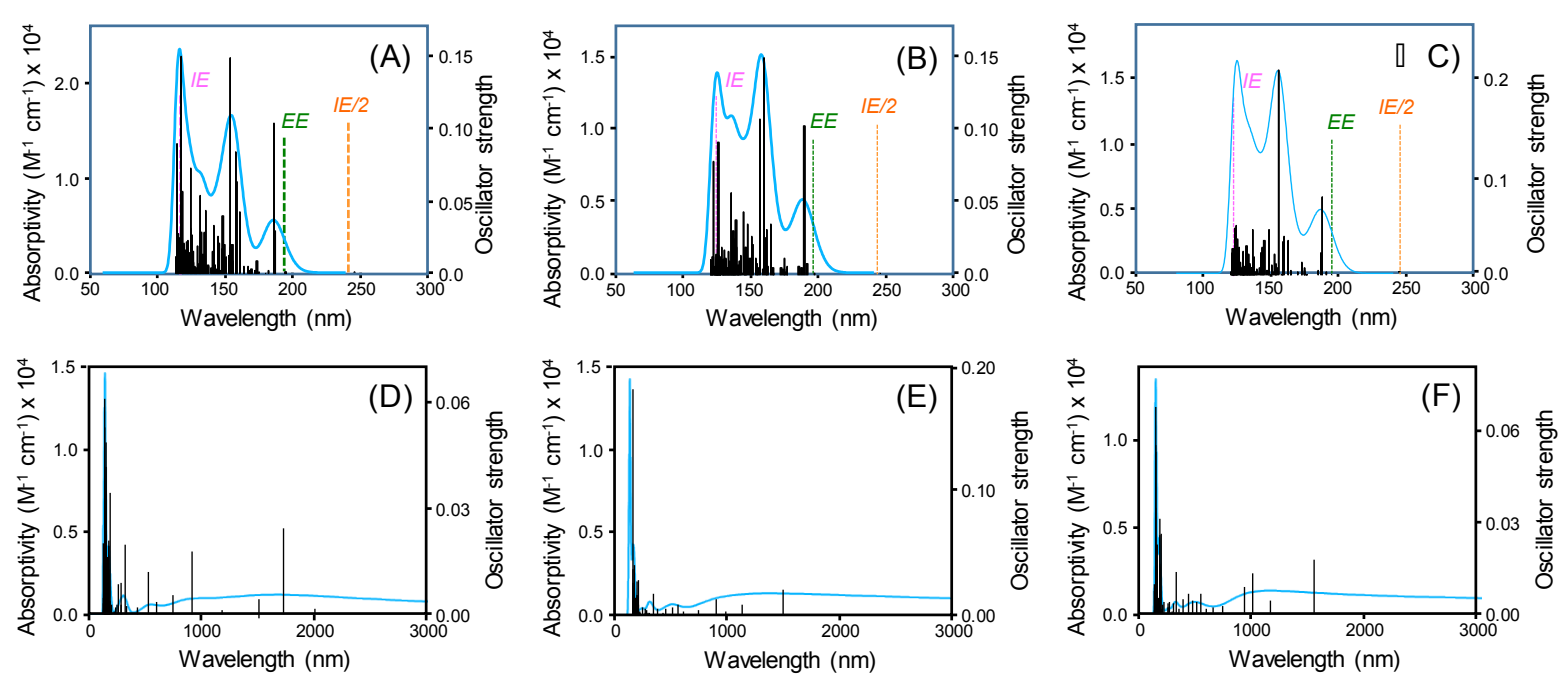

Fig. S20. Absorption spectra calculated for non-Mirzayanov's Novichok. (A) - (C) absorption spectra for neutral species. (D) - (F) absorption spectra for singly-charged ions. (A) (D) A230 (B) (E) A232 (C) (F) A234. 


\section{References}

(S1) Wikipedia, "Stockholm Convention on Persistent Organic Pollutants"; https://en.wikipedia.org/wiki/ Stockholm Convention on Persistent Organic Pollutants.

(S2) Wikipedia, "Novichok agent”; https://en.wikipedia.org/wiki/Novichok agent.

(S3) World News on 16 Mar., 2018. OPCW: No member states are known to possess 'Novichok-class' nerve agents: https://www.rt.com/news/421512-opcw-no-member-states-novichok/.

(S4) Mirzayanov, V. S. State Secrets. An Insider's Chronicle of the Russian Chemical Weapons Program. (2009) pp. 142-145, 179-180, ISBN 978-1-4327-2566-2.

(S5) Chen, B. H.; Liu, J. T.; Chen, W. X.; Chen, H. M.; Lin, C. H. A general approach to the screening and confirmation of tryptamines and phenethylamines by mass spectral fragmentation. Talanta 74, 512-517 (2008).

(S6) Hosseini, S. E.; Saeidian, H.; Amozadeh, A.; Naseri, M. T.; Babri, M. Fragmentation pathways and structural characterization of organophosphorus compounds related to the Chemical Weapons Convention by electron ionization and electrospray ionization tandem mass spectrometry. Rapid Commun. Mass Spectrom. 30, 2585-2593 (2016).

(S7) Son, V. V.; Nakamura, H.; Imasaka, T.; Imasaka, T. Determination of nerve agent metabolites in human urine by femtosecond laser ionization mass spectrometry using 2-(bromomethyl)naphthalene as a derivatizing reagent. Anal. Chim. Acta 1069, 82-88 (2019).

(S8) Matsui, T.; Fukazawa, K.; Fujimoto, M.; Imasaka, T. Analysis of persistent organic pollutants at subfemtogram levels using a high-power picosecond laser for multiphoton ionization in conjunction with gas chromatography/time-of-flight mass spectrometry. Anal. Sci. 28, 445-450 (2012). 